\title{
Cytochalasin B-induced triploidy in mouse oocytes fertilized in vitro
}

\author{
Anna Niemierko and Aldona Komar \\ Laboratory of Experimental Embryology, Institute of Obstetrics and Gynaecology, \\ 00-315 Warsaw, Karowa 2, Poland
}

\begin{abstract}
Summary. Mouse eggs and spermatozoa were treated in various ways with 5 or $10 \mu \mathrm{g}$ cytochalasin $\mathrm{B} / \mathrm{ml}$. The fertilization rate in vitro was reduced by treatment with the drug but $80-90 \%$ of the eggs fertilized were triploid. Many of the experimental eggs were penetrated by one or more spermatozoa but remained unfertilized $(75 \%$ compared with $9 \%$ in control eggs). It is suggested that cytochalasin $\mathbf{B}$ weakens the zona reaction and interferes with fusion of gametes but does not prevent the block to polyspermy.
\end{abstract}

\section{Introduction}

Information on the developmental effects of triploidy in the mouse, especially in the postimplantation period, is still scarce, and progress in this field depends on the development of an efficient technique which would permit the production of a large number of triploid embryos. Of the various agents which have been used in the past to induce this aberration, the most promising appears to be cytochalasin B (Niemierko, 1975). Mouse eggs fertilized in vivo and treated with this drug in vitro at the time of the second meiotic division react by suppression of the second polar body which leads to digynic triploidy. The aim of the present study was to explore further this approach using an in-vitro system and to analyse the effect of cytochalasin $B$ on mouse gametes and the process of fertilization.

\section{Materials and Methods}

The eggs were obtained from Swiss albino females from a randomly bred colony. The females were induced to superovulate by i.p. injections of 5 i.u. PMSG and 5 i.u. HCG given $43-48 \mathrm{hr}$ apart and killed 13-17 hr (mostly $13 \mathrm{hr}$ ) after the second injection. Fertilization in vitro was carried out according to the method of Toyoda, Yokoyama \& Hosi (1971). Spermatozoa were obtained from the cauda epidydymidis and vas deferens of $F_{1}(\mathrm{CBA} / \mathrm{H} \times \mathrm{C} 57 \mathrm{BL})$ males and suspended in $0.4 \mathrm{ml}$ medium (Toyoda et al., 1971). Part of this suspension $(0.04 \mathrm{ml}$ ) was added to eggs kept in $0.4 \mathrm{ml}$ medium under liquid paraffin.

Four experiments were carried out to examine the effect of cytochalasin B on gametes and to evaluate the optimal conditions for inducing triploidy in vitro. In Exp. I, eggs were fertilized and cultured for $7 \mathrm{hr}$ in the presence of 10 or $5 \mu \mathrm{g}$ cytochalasin B/ml. In Exp. II, eggs were inseminated for 2 or $2 \frac{1}{2} \mathrm{hr}$ in cytochalasin B-free medium and transferred for 5 or $4 \frac{1}{2} \mathrm{hr}$ to medium containing $10 \mu \mathrm{g}$ cytochalasin B/ml. In Exp. III the eggs were pretreated with cytochalasin B $(10 \mu \mathrm{g} / \mathrm{ml})$ for $1 \frac{1}{2}$ $\mathrm{hr}$, and then washed for a long $(1 \mathrm{hr})$ or short $(1 \mathrm{~min})$ period before insemination in cytochalasin B-free medium. In Exp. IV spermatozoa were pretreated with cytochalasin B $(10 \mu \mathrm{g} / \mathrm{ml})$ for $1 \mathrm{hr}$, before use for insemination in cytochalasin B-free medium.

Each experiment was as a rule accompanied by control fertilization using gametes obtained from the same animals. Control gametes received the same treatment (cytochalasin B-free) as the experimental gametes (Control I). We also collected data on fertilization in vivo in Swiss albino females induced to ovulate and mated with $\mathrm{F}_{1}(\mathrm{CBA} / \mathrm{H} \times \mathrm{C} 57 \mathrm{BL})$ males (Control II).

Each experiment lasted for $7 \mathrm{hr}$, and then the eggs were fixed and mounted as permanent preparations by the method of Mulnard (1955), as modified by Tarkowski \& Wróblewska (1967). 


\section{Results}

Experiment $I$. As shown in Table 1, fertilization rate was affected by a high concentration of cytochalasin B in the medium. However, at both concentrations, the incidence of triploidy of the fertilized eggs was similar. Neither concentration was fully effective in suppressing the formation of the second polar body because in both there were diploid eggs.

Experiment II. The results of avoiding the adverse effect of cytochalasin B on gametes at the very time of fertilization by inseminating eggs in cytochalasin B-free medium before incubation with it are shown in Table 2 . Fertilization rate after $2 \mathrm{hr}$ was low, but had improved by $2 \frac{1}{2} \mathrm{hr}$. The incidence of triploidy was again very high. These results confirm those of Exp. I that cytochalasin B at a concentration of $10 \mu \mathrm{g} / \mathrm{ml}$ affects the fertilization process and show that the peak of penetration of spermatozoa into eggs does not occur until at least $2 \frac{1}{2} \mathrm{hr}$ after mixing the gametes.

Experiments III and $I V$. These were carried out to determine whether cytochalasin $\mathrm{B}$ at the $10 \mu \mathrm{g} / \mathrm{ml}$ concentration affects the eggs or spermatozoa and whether it permanently decreases the ability of gametes to complete fertilization. The results of Exp. III (Table 3) showed that long washing eliminated to a high degree the adverse effect of cytochalasin B. After both treatments diploid eggs predominated. The results of Exp. IV (Table 4) showed that the fertilizing ability of spermatozoa pretreated with cytochalasin $B$ was significantly reduced. The degree of agglutination of the spermatozoa was similar in the two media, and cytochalasin B did not impair the motility of the spermatozoa. For technical reasons it was not possible to wash the spermatozoa and consequently some cytochalasin $\mathrm{B}$ was introduced into the fertilization medium, the final concentration being about 0.9 $\mu \mathrm{g} / \mathrm{ml}(0.04 \mathrm{ml}$ medium containing $10 \mu \mathrm{g}$ cytochalasin $\mathrm{B} / \mathrm{ml}$ mixed with $0.4 \mathrm{ml}$ clean medium). Such a concentration is probably too low to have a significant effect on fertilization because $5 \mu \mathrm{g} / \mathrm{ml}$ only slightly reduces the rate of fertilization (Table 1). It appears, therefore, that the low fertilization rate in this experiment was the result of a decrease in the fertilizing ability of the spermatozoa treated with cytochalasin B rather than of the presence of traces of the substance in the fertilization medium. However, the amount of cytochalasin B introduced into this medium was sufficient to suppress extrusion of the second polar body in some of the eggs because the incidence of triploidy was increased (Table 4).

Penetration of eggs by supernumerary spermatozoa. A large number of eggs fertilized in vitro were penetrated by supplementary spermatozoa (Tables $1-4$ ) but polyspermy was relatively rarely observed. The frequency of eggs with supernumerary spermatozoa in the perivitelline space was significantly higher after some experimental treatments than in the controls. A high proportion (75\%) of eggs was penetrated by more than one spermatozoon but remained unfertilized.

Anomalies of fertilization and egg maturation in control experiments. It would be meaningless to calculate an overall fertilization rate since the results of control experiments varied substantially in the different experiments. Consequently these results are presented separately in Tables 1-4, each describing one experimental series. However, to assess properly the effect of treating the gametes with cytochalasin $B$, the type and frequency of various anomalies in control material is of importance.

In the Control I series, among 522 eggs fertilized in vitro there were 15 dispermic triploids $(1.6 \%)$, 3 digynic triploids $(0.3 \%), 1$ trispermic tetraploid $(0 \cdot 1 \%)$ and 4 eggs which underwent immediate cleavage $(n / 2 n$ mosaics $-0.4 \%)$.

In the Control II series, of 389 eggs collected from 15 females induced to superovulate and mated 195 eggs were fertilized (50.1\%). The only anomalous eggs were 3 triploid eggs $(0 \cdot 8 \%)$, of which 2 were dispermic and one digynic.

\section{Discussion}

In the present experiments, treatment with cytochalasin B was combined with fertilization of mouse eggs in vitro, rather than in vivo as in previous work (Niemierko, 1975), and was found to be a convenient method of producing large numbers of triploid eggs. A high percentage ( $80-90 \%$ ) of triploid eggs was obtained when eggs were subjected to 5 or $10 \mu \mathrm{g}$ cytochalasin $\mathrm{B} / \mathrm{ml}$ for 2 or $2 \frac{1}{2} \mathrm{hr}$ after 


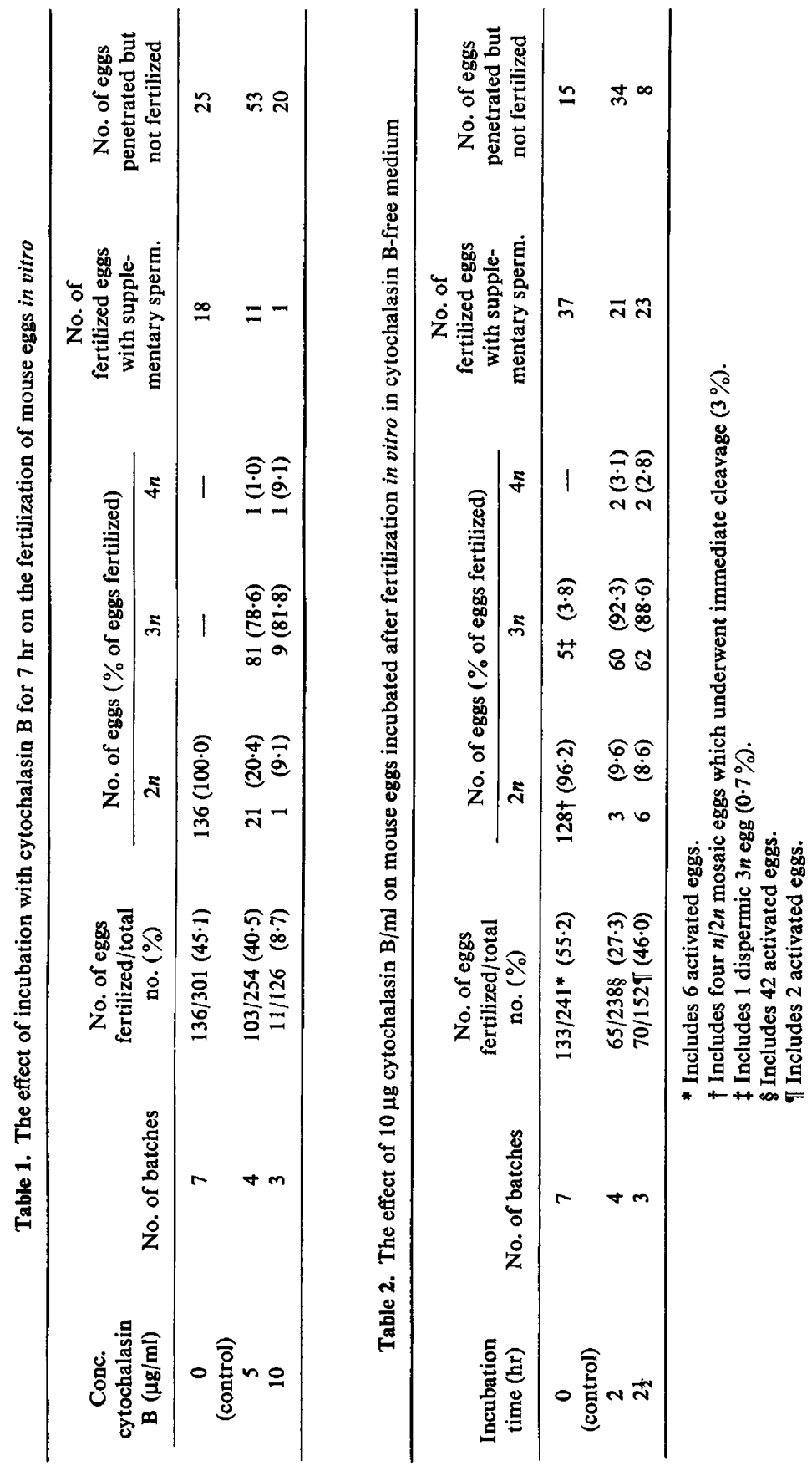




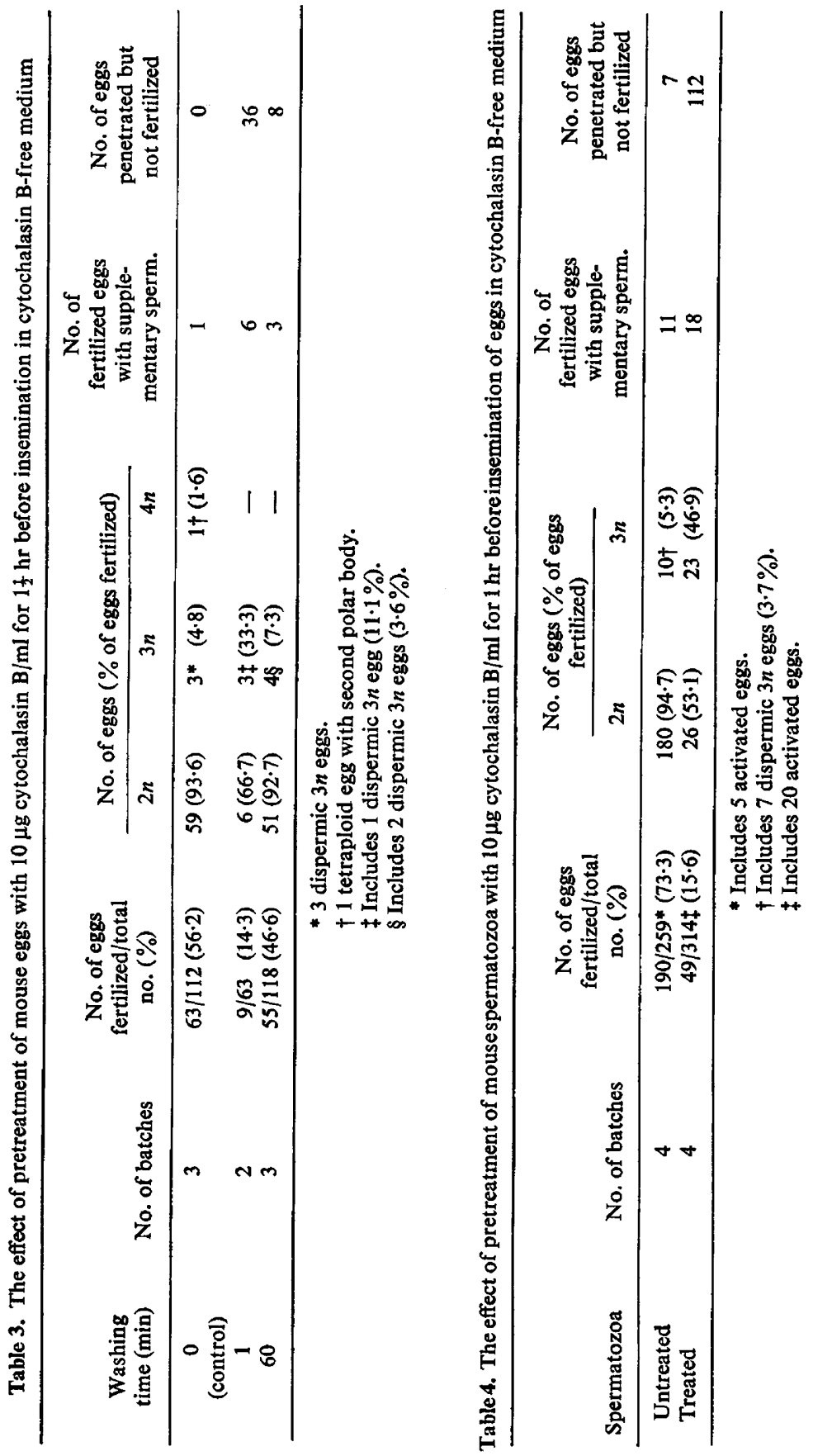


insemination in cytochalasin B-free medium, even when the fertilization rate was low. The in-vivo method of Niemierko (1975) was laborious and time consuming because of the limited number of matings available simultaneously and because the eggs needed to be subjected to cytochalasin $\mathbf{B}$ at the precise time of extrusion of second polar body. This condition could not be fully met because the process of fertilization in any female is extended and only a few eggs are at a suitable stage at any one moment. The advantage of the present procedure consists, therefore, in the overall efficiency of suppressing the second polar body and in its simplicity.

It is known that the rate of fertilization in vitro and in vivo depends, among other factors, on the genotype of the gametes (Krzanowska, 1964, 1970; Whittingham, 1968; Iwamatsu \& Chang, 1971; S. Kaleta, personal communication). In the genetic combination of gametes employed in the present experiments (Swiss albino eggs, $F_{1}(\mathrm{CBA} / \mathrm{H} \times \mathrm{C} 57 \mathrm{BL})$ spermatozoa), the proportion of eggs fertilized in vitro and in vivo (Control series I and II) was low, about $50 \%$. Because the yield of triploid eggs depends on the overall rate of fertilization, the efficiency of this method could probably be increased by employing gametes of other genotypes which give a higher percentage of fertilization.

Treatment of mouse eggs with cytochalasin B appears to affect fertilization because it weakens the zona reaction. This conclusion is supported by the fact that the incidence of eggs with supernumerary spermatozoa and the mean number per egg of spermatozoa which have penetrated the zona pellucida were consistently higher, often significantly so, in experimental than in control eggs. However, despite this increase in the penetration of spermatozoa into experimental eggs the rate of polyspermic fertilization was low and similar to that in the controls (about $2 \%$ ). This suggests that cytochalasin B does not affect the block to polyspermy at the level of the plasma membrane in mouse eggs, although Ziomek \& Epel (1975) found that it did so in the eggs of a snail, Spisula solidissima.

The high incidence of penetrated but unfertilized eggs in Exps III and IV suggests that cytochalasin $B$ also interferes with the fusion of gametes. Since fertilization involves fusion of the membranes of the gametes, it is very probable that this substance, especially at high concentrations, impairs fusion by changing the architecture of the cell membrane of eggs and spermatozoa. Everhart \& Rubin (1974) used the scanning electron microscope to establish that cytochalasin $B$ altered the fine structure of the cell surface and that the degree of change partly depended on the concentration of cytochalasin B. Johnson, Eager, Muggleton-Harris \& Grave (1975) showed that cytochalasin B changed the pattern of fluorescence of Concanavalin A on unfertilized mouse eggs, and interpreted this as a corrugation of the membrane. Such changes in the acrosomal region of the spermatozoon might impair release of enzymes and consequently prevent fertilization. Changes in the postacrosomal region and in the plasma membrane of the oocyte might obstruct fusion of the gametes. Cytochalasin B is known to block many cellular processes which involve fusion of membranes, e.g. pinocytosis, phagocytosis, secretion, cytokinesis, Sendai virus-mediated fusion of cells (Pasternak \& Micklem, 1973; Ahkong, Fischer, Tampion \& Lucy, 1975), and the in-vitro system described in this paper may be useful for investigating this aspect of its action.

We thank Professor A. K. Tarkowski for his advice and critical reading of the manuscript.

\section{References}

Ahkong, Q.F., Fischer, D., TAmPion, W. \& LuCY, J.A. (1975) Mechanism of cell fusion. Nature, Lond. 253, 194-195.

EVERHART, L.P. \& RuBRN, R.W. (1974) Cyclic changes in the cell surface. II. The effect of cytochalasin $B$ on the surface morphology of synchronized Chinese hamster ovary cells. J. Cell Biol. 60, 442-447.

Iwamatsu, T. \& ChaNG, M.C. (1971) Factors involved in the fertilization of mouse eggs in vitro. J. Reprod. Fert. 26, 197-208.

Johnson, M.H., EAger, D., Muggleton-HarRis, A. \&
Grave, H.M. (1975) Mosaicism in organisation of Concanavalin A receptors on surface membrane of mouse egg. Nature, Lond. 257, 321-322.

Krzanowska, H. (1964) Time interval between copulation and fertilization in inbred lines of mice and their crosses. Folia biol., Krakbw 12, 231-244.

KrzanowsKa, H. (1970) Relation between fertilization rate and penetration of eggs by supplementary spermatozoa in different mouse strains and crosses. J. Reprod. Fert. 22, 199-204.

Mulnard, J. (1955) Contribution à la connaissance 
dans l'ontogénèse. Les phosphomonoestérases acide et alcaline dans le dévelopment du Rat et de la Souris. Archs Biol. 66, 525-685.

NiEmiERKo, A. (1975) Induction of triploidy in the mouse by cytochalasin B. J. Embryol. exp. Morph. 34, 279289.

Pasternak, C.A. \& Micklem, K.J. (1973) Permeability changes during cell fusion. J. Membrane Biol. 14, 293-303.

Tarkowski, A.K. \& Wróblewska, J. (1967) Development of blastomeres of mouse eggs isolated at the 4- and 8-cell stage. J. Embryol. exp. Morph. 18, 155180.

Toyoda, Y., Yokoyama, M. \& Hosi, T. (1971) Studies on the fertilization of mouse eggs in vitro. I. In vitro fertilization of eggs by fresh epididymal sperm. Jap. J. Anim. Reprod. 16, 147-151.

WhITTINGHAM, D.G. (1968) Fertilization of mouse eggs in vitro. Nature, Lond. 220, 592-593.

ZIomeK, C.A. \& EPEL, D. (1975) Polyspermy block of Spisula eggs is prevented by cytochalasin B. Science, N. Y. 189, 139-142.

Received 5 March 1976 